\title{
The Gerasimov-Drell-Hearn Sum Rule and the Spin Structure of the Proton
}

\author{
G. Krein \\ Instituto de Física Teórica, Universidade Estadual Paulista, \\ Rua Pamplona 145, 01405-900, São Paulo, SP
}

Received on 22 February, 2001

\begin{abstract}
We present an overview of the present theoretical status of the Gerasimov-Drell-Hearn (GDH) sum rule. It is emphasized the importance of near threshold $s$-wave photoproduction contributions to the sum rule. We also discuss the experimental verification of the sum rule, with particular emphasis on the recent experiment at the Mainz Microtron (MAMI). The spin polarizabilities and the generalized GDH sum rule, which are of interest to issues related to the spin of the proton as measured at high energies, are discussed.
\end{abstract}

\section{Introduction}

The response of the internal degrees of freedom of the nucleon to an external electromagnetic field can be described in terms of polarizabilities. For real photons, polarizabilities and other ground-state properties can be related to integrals over photoabsorption cross sections by sum rules. One of the most prominent examples is the Gerasimov-Drell-Hearn (GDH) sum rule [1], which provides a relationship between the anomalous magnetic moment $\kappa_{N}$ of the nucleon and the photoabsorption cross sections for parallel and antiparallel alignments of the nucleon and photon helicities, $\sigma_{3 / 2}$ and $\sigma_{1 / 2}$

$$
-\frac{\kappa_{N}^{2}}{4}=\frac{M^{2}}{8 \pi^{2} \alpha} I_{N}
$$

where $I_{N}$ is the GDH integral, given by

$$
I_{N}=\int_{\omega_{t h}}^{\infty} \frac{\sigma_{1 / 2}(\omega)-\sigma_{3 / 2}(\omega)}{\omega} d \omega .
$$

The importance of the sum rule stems from the fact that it is based on general principles of physics, such as Lorentz and gauge invariance, crossing symmetry, causality and unitarity.

Closely related to the GDH sum rule is the forward spin polarizability [2]

$$
\gamma=\frac{1}{4 \pi^{2}} \int_{\omega_{t h}}^{\infty} \frac{\sigma_{1 / 2}(\omega)-\sigma_{3 / 2}(\omega)}{\omega^{3}} d \omega
$$

This quantity is related to the Ragusa polarizabilities [3] $\gamma_{i}, i=1 \cdots 4$, as

$$
\gamma=\gamma_{1}-\gamma_{2}-2 \gamma_{4}
$$

The Ragusa polarizabilities appear as low-energy contants of the terms $\mathcal{O}\left(\omega^{3}\right)$ in the expansion of the Compton amplitude in powers of the photon energy $\omega$.
The use of virtual photons from electron scattering processes provides us with even more detailed information on the internal degrees of freedom of the nucleon. In particular, as we increase the four-momentum $Q^{2}$ of the virtual photon from the real-photon point $\left(Q^{2}=0\right)$ to large values of $Q^{2}$, we can investigate the transition from the nonperturbative to the perturbative regime of quantum chromodynamics (QCD). The generalizations of real-photon sum rules to virtual photons provide an interesting possibility to study this transition and the varying role of the relevant degrees of freedom.

In this contribution we present an overview of the present theoretical and experimental status of the GDH sum rule. We also discuss generalisations of the spin polarizability and GDH sum rule. These generalizations are of interest to issues related to the spin of the proton as measured at high energies. At the end of this contribution, we also discuss a generalization of the Baldin sum rule as a function of $Q^{2}$ and compare predictions based on chiral perturbation theory and a phenomenological resonance model.

\section{The GDH sum rule}

Until recently, there was no direct measurement of the cross sections $\sigma_{3 / 2}$ and $\sigma_{1 / 2}$ in the integrands of the integrals above. The situation changed with the recent experiment at MAMI [4], which measured the crosssection difference $\Delta \sigma=\sigma_{1 / 2}^{N}-\sigma_{3 / 2}^{N}$ for photon lab. energies in the range $200-800 \mathrm{MeV}$. Before this experiment, one had to rely on estimates using pion photoproduction amplitudes for $\Delta \sigma$, as compiled, for example, by the SAID group [5]. 
The decomposition of $\Delta \sigma$ in Eqs. (2) and (3) in terms of photoproduction multipoles is given as [6]

$$
\begin{aligned}
\Delta \sigma & =8 \pi \frac{q}{k}\left(\left|E_{0+}\right|^{2}+3\left|E_{1+}\right|^{2}+6 E_{1+}^{*} M_{1+}-\left|M_{1+}\right|^{2}\right. \\
& \left.+\left|M_{1-}\right|^{2}+\cdots\right),
\end{aligned}
$$

where $q$ is the c.m. momentum of the pion and $k$ in the c.m. momentum of the photon. Because of the weighting factor $1 / \omega$ in $I_{G D H}$, it is to be expected that a large fraction of the the sum rule is saturated by $s$-wave nearthreshold pion photoproduction and by $\Delta(1232)$ resonance production. This seems particularly true for $\gamma$, because of the $1 / \omega^{3}$ in the integrand of Eq. (3).

The status of the GDH sum rule until recently was, as discussed in Refs. [7] and [8], that there seemed to be a severe discrepancy between the prediction of the sum rule and the saturation of the integral with photoproduction data. For the proton, for example, the sum rule predicts

$$
I_{p}=-204 \mu \mathrm{b}
$$

while the SAID multipoles, together with an old estimate [9] of the $\pi N N$ contribution, predicted [7] $I_{p}=$ $-289 \mu \mathrm{b}$. A more recent SAID analysis, SAID-SP97K, gave $[8]$

$$
I_{p}^{S A I D}=-281 \mu \mathrm{b}
$$

The discrepancy between the prediction of the sum rule and the SAID multipoles was on the order of $30 \%$.

Very recently, the present author together with D. Drechsel [10] showed that the discrepancy is drastically reduced when using the HDT [11] multipoles, instead of the SAID multipoles. The HDT multipoles are based on fixed- $t$ dispersion relations and unitarity. In the HDT dispersive approach the threshold region is not included in the fitting of free parameters; the threshold values for the $s$-wave amplitudes are genuine predictions. The HDT analysis is limited to photon energies up to $400 \mathrm{MeV}, s, p$, and $d$ waves for isospin $1 / 2$, and $s$ and $p$ waves for isospin $3 / 2$. Although the differences between the SAID-SP97K and HDT multipoles are very small for energies above $400 \mathrm{MeV}$, large differences occur for the $E_{0+}$ multipole for $\pi^{+}$production close to threshold. In Fig. 1 we show the results for the integrand of the sum rule using the HDT (solid) and SAID (dashed) multipoles.
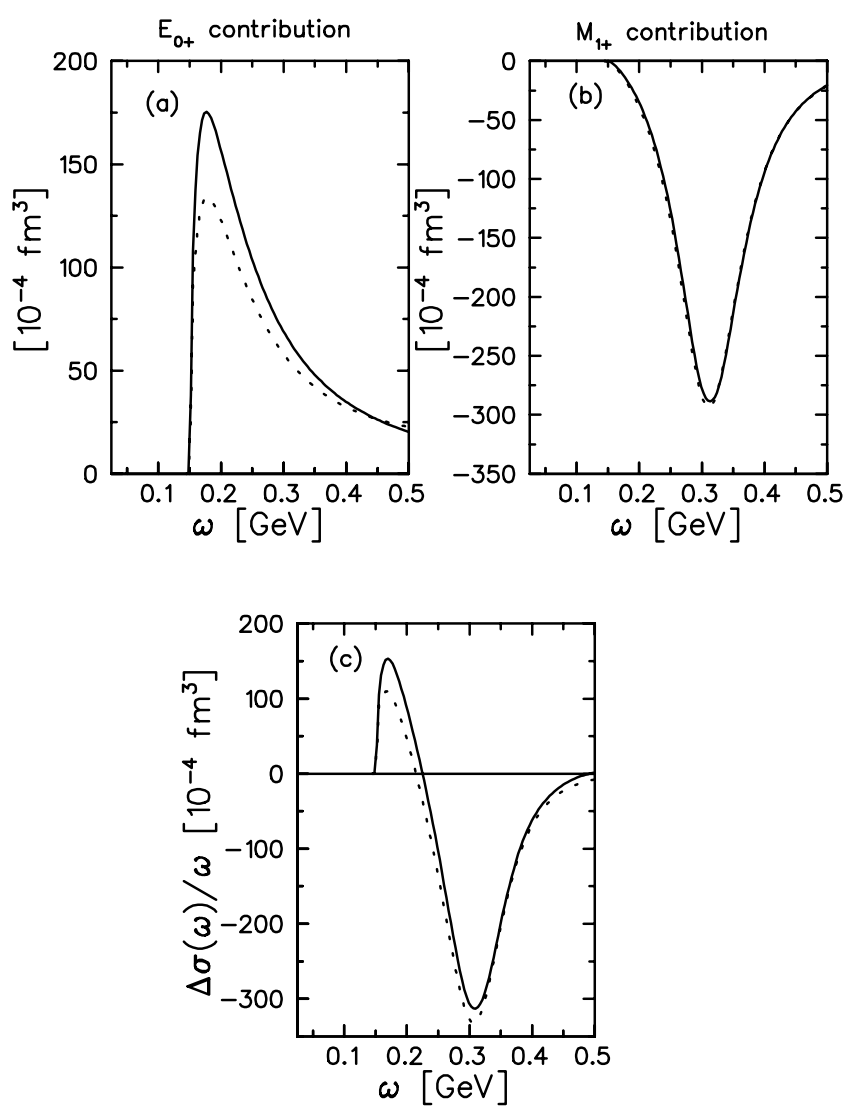

Figure 1. Multipole contributions to the integrand of $I_{p}$ : (a) $E_{0^{+}}$, (b) $M_{1+}$, and (c) all (up to $L=5$ ) partial waves. The solid lines correspond to the HDT multipoles and the dotted to the SP97K-SAID multipoles.

The HDT multipoles lead to $I_{p}(1 \pi)=-196 \mu \mathrm{b}$. This implies in a change of $20 \mu \mathrm{b}$, compared to SAID. If we use the (very) old estimate estimate of Karliner [9] for the two-pion contribution, $I_{p}(2 \pi)=-65 \mu \mathrm{b}$, the earlier discrepancy [7] reduces from $38 \%$ to $28 \%$. Moreover, if one uses the estimate of Ref. [12] of the contributions beyond one-pion production, $-32 \mu \mathrm{b}$, together with the single-pion HDT prediction, the discrepancy falls to only $12 \%$.

This value is not too far the result of the recent GDH experiment at Mainz [4], for energies up to $800 \mathrm{MeV}$. Concerning the remaining discrepancy, it will be interesting to see the outcome of the GDH experiment at ELSA at higher energies.

Regarding the spin polarizability, Eq. (3), because of the $1 / \omega^{3}$ in the denominator, one expects that nearthreshold $s$-wave pion photoproduction and $\Delta(1232)$ resonance production saturate the above integral even more rapidly than the GDH integral. This was actually verified in Ref. [13]: the integrand is already negligible at $\omega \simeq 500 \mathrm{MeV}$.

The HDT multipoles lead for the proton

$$
\gamma_{p}^{H D T}=-0.6 \times 10^{-4} \mathrm{fm}^{4},
$$

while the SP97K-SAID give

$$
\gamma_{p}^{S A I D}=-1.3 \times 10^{-4} \mathrm{fm}^{4} .
$$


The recent Mainz experiment [4] of $\Delta \sigma=\sigma_{1 / 2}-\sigma_{3 / 2}$ for energies larger than $200 \mathrm{MeV}$ gives a value of $\gamma_{p} \sim$ $-1.7 \times 10^{-4} \mathrm{fm}^{4}$. The HDT multipoles predict that the region very close to threshold, $\omega_{t h}<\omega<200 \mathrm{MeV}$, contributes to $\gamma_{p}$ with a positive value equal to +0.9 . Therefore, to the extent that the HDT multipoles provide an accurante description of the low-energy region, one can predict that $\gamma_{p}=-0.8 \times 10^{-4} \mathrm{fm}^{4}$.

It is instructive to compare [13] these numbers with predictions of chiral perturbation theory. Two recent calculations, Refs. [14] and [15], in $\mathcal{O}\left(p^{4}\right)$ of HBChPT find $\gamma_{p}=-3.9 \times 10^{-4} \mathrm{fm}^{4}$. Another recent $\mathcal{O}\left(p^{4}\right)$ calculation [16], which differs from the previous two in the way the single-particle reducible contributions are treated, leads to $\gamma_{p}=-1.0 \times 10^{-4} \mathrm{fm}^{4}$. A calculation [17] using dispersion relations together with large $N_{c}$ arguments leads to $\gamma_{p}=-0.1 \times 10^{-4} \mathrm{fm}^{4}$.

\section{Generalized GDH integral and spin polarizability}

One of the many possible generalizations of the GDH sum rule [18] to virtual photons is the expression

$$
\begin{aligned}
I_{1}\left(Q^{2}\right) & =\frac{M^{2}}{8 \pi^{2} \alpha} \int_{\nu_{0}}^{\infty} \frac{1-x}{1+\gamma^{2}}\left[\sigma_{1 / 2}\left(\nu, Q^{2}\right)-\sigma_{3 / 2}\left(\nu, Q^{2}\right)\right. \\
& \left.-2 \gamma \sigma_{L T}^{\prime}\left(\nu, Q^{2}\right)\right] \frac{d \nu}{\nu}
\end{aligned}
$$

where $\nu_{0}=m_{\pi}+\left(m_{\pi}^{2}+Q^{2}\right) / 2 m$ is the threshold $l a b$ energy of one-pion production. Here, $x_{0}$ is the threshold value of $x=Q^{2} / 2 M \nu, x_{0}=Q^{2} /\left(M m_{\pi}+m_{\pi}^{2}+Q^{2}\right)$, and $\gamma=Q / \nu$. Also, $\sigma_{3 / 2}$ and $\sigma_{1 / 2}$ are the cross sections for the scattering of polarized electrons on polarized nucleons with parallel and antiparallel alignments of the electron and nucleon helicities, and $\sigma_{L T}^{\prime}$ is the longitudinal-transverse cross section.

Contact with deep inelastic scattering experiments can be made by expressing $I_{1}\left(Q^{2}\right)$ in terms of an integral over the spin structure function $g_{1}\left(x, Q^{2}\right)$

$$
\begin{aligned}
I_{1}\left(Q^{2}\right) & =\frac{2 M^{2}}{Q^{2}} \int_{\nu_{0}}^{x_{0}} g_{1}\left(x, Q^{2}\right) d x \\
& =\frac{2 M^{2}}{Q^{2}} \Gamma_{1}\left(Q^{2}\right) .
\end{aligned}
$$

A generalized polarizability can also be defined in terms of electroproduction cross sections $\sigma_{3 / 2}$ and $\sigma_{1 / 2}$ as [19]

$$
\gamma\left(Q^{2}\right)=\frac{1}{4 \pi^{2}} \int_{\nu_{0}}^{\infty} \frac{\sigma_{1 / 2}\left(\nu, Q^{2}\right)-\sigma_{3 / 2}\left(\nu, Q^{2}\right)}{\nu^{3}} d \nu
$$

There is very little experimental information on the electroproduction cross-sections in the resonance region. The situation will change in the near future with the new data from the Jefferson Lab. For the time being, one has to rely on phenomenological models. Here, we review our recent work [20] [21] on generalized GDH sum rule and spin polarizabilities. In these works we used the recently developed isobar model for electroproduction, known as MAID. The model is well documented and accessible via the Internet [22]. It is based on an effective phenomenological Lagrangian for Born terms ("background") and resonances up to the third resonance region. For each partial wave the multipoles satisfy the constraints of gauge invariance and unitarity, and in the real photon case the results agree well with the predictions of dispersion theory [11]. The model is able to describe the correct energy dependence of the multipoles for photon energies up to $\omega \simeq 1 \mathrm{GeV}$, and it provides a good description of all experimentally measured differential cross sections and polarization observables.

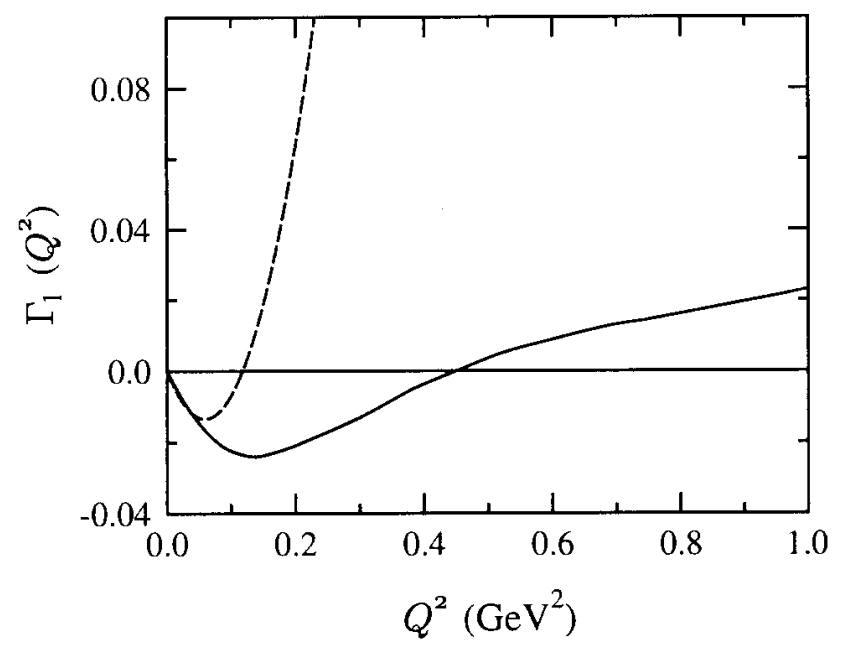

Figure 2. Comparison of results for $\Gamma_{1}$ from MAID (solid) and HBChPT (dashed).

The prediction of MAID is showm in Fig. ??. We compare results with a recent $\mathrm{HBChPT}$ calculation [23] at $\mathcal{O}\left(p^{4}\right)$. For the proton, the result obtained in Ref. [23] for $\Gamma_{1}$ is given by

$$
\Gamma_{1}\left(Q^{2}\right)=-\frac{\kappa_{p}}{4}+6.85 Q^{2}\left(\mathrm{GeV}^{2}\right)
$$

Being this a result of a HBChPT calculation, it is expected to be valid only for small values of $Q^{2}$. In the Figure we compare the predictions of MAID (solid line) and Eq. (13) (dashed line). Is is woth mentioning the the MAID result is very close to recent SLAC data [20] at $Q^{2}=0.5 \mathrm{GeV}^{2}$. From the figure, it is seen that for $Q^{2}>0.05 \mathrm{GeV}^{2}$, the HBChPT result becomes considerably different from the MAID prediction.

For the generalized spin polizability, a recent calculation [19] has been made in the framework of the oneloop approximation to (relativistic) chiral perturbation theory (ChPT), supplemented by tree graphs for the excitation of the $\Delta(1232)$ resonance in the relativistic Rarita-Schwinger formalism. 


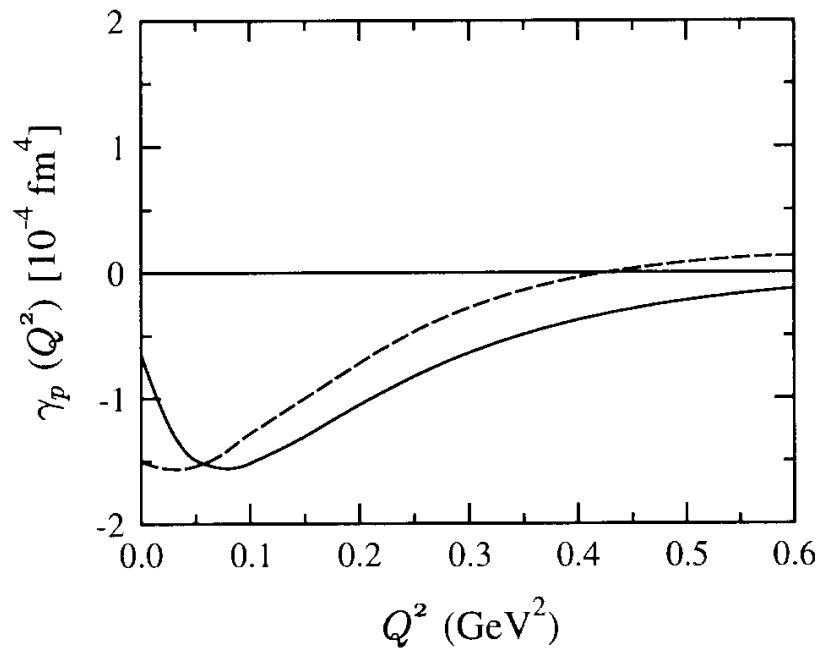

Figure 3. Comparison of results for $\gamma_{p}$ from MAID (solid) and relativistic ChPT (dashed).

In Fig. 3 we compare results again with MAID [21]. The most striking difference refers to the slope of $\gamma_{p}\left(Q^{2}\right)$ close to the real photon point. The pronounced slope in $\gamma_{p}\left(Q^{2}\right)$ observed in the MAID result is due to the interference between background and $\Delta(1232)$ terms, which has its the physical origin in the dynamical dressing of the $\gamma N \Delta$ vertex [24].

In summary, one of the striking features of the generalized GDH integral is its rapid fluctuation with $Q^{2}$ and in particular a change of sign at $Q^{2} \simeq 0.5(\mathrm{GeV})^{2}$, which imposes severe constraints on any model for the nucleon structure. This zero-crossing separates the region dominated by resonance-driven coherent processes from a region of essentially incoherent scattering off the nucleon's constituents. A similar zero-crossing is predicted by ChPT for the generalized spin polarizability, $\gamma\left(Q^{2}\right)$ [19], while we found that MAID excludes such a cross-over for $Q^{2} \leq(1 \mathrm{GeV})^{2}$.

Acknowledgments Work partially supported FAPESP and CNPq.

\section{References}

[1] S.B. Gerasimov, Sov. J. Nucl. Phys. 2, 430 (1966); S.D. Drell and A.C. Hearn, Phys. Rev. Lett. 16, 908 (1966).

[2] M. Gell-Mann, M. Goldberger and W. Thirring, Phys. Rev. 95, 1612 (1954).

[3] S. Ragusa, Phys. Rev. D 47, 3757 (1993); Phys. Rev. D 49, 3157 (1994).
[4] J. Ahrends er al. (GDH Collaboration), Phys. Rev. Lett. 84, 5904 (2000).

[5] SAID: The Scattering Analysis Interactive Dial-in program, data available at http://gwdac.phys.gwu.edu .

[6] For a review see: D. Drechsel, Prog. Part. Nucl. Phys. 34, 181 (1995).

[7] A.M. Sandorfi, C.S. Whisnant, and M. Khandaker, Phys. Rev. D 50, R6681 (1994).

[8] R.A. Arndt, I.I. Strakovsky, and R. Workman, Phys. Rev. C 53, 430 (1996).

[9] I. Karliner, Phys. Rev. D 7, 2717 (1973).

[10] D. Drechsel and G. Krein, Phys. Rev. D 58, 116009, (1998).

[11] O. Hanstein, D. Drechsel, and L. Tiator, Phys. Lett. B 385, 45 (1996); Nucl. Phys. A 399, 13 (1996); Nucl. Phys. A 632, 561 (1998).

[12] V. Burkert and Z. Li, Phys. Rev. D 47, 46 (1993).

[13] D. Drechsel, G. Krein and O. Hanstein, Phys. Lett. B 420, 248 (1998).

[14] X. Ji, C.-W. Kao and J. Osborne, Phys. Rev. D 61, 074003 (2000).

[15] K.B.V. Kumar, J.A. McGovern and M. C. Birse, Phys. Lett. B 479, 167 (2000).

[16] G.C. Gellas, T.R. Hemmert and Ulf-G. Meissner, Phys. Rev. Lett. 85, 14 (2000).

[17] Y. Tanushi, S. Saito, M. Uehara, Phys. Rev. C 60, 065213 (1999).

[18] R. Pantförder, Investigations on the Foundation and Possible Modifications of the Gerasimov-DrellHearn Sum Rule, Ph.D. Thesis, Bonn (1997); hepph/9805434.

[19] J. Edelmann, N. Kaiser, G. Piller and W. Weise, Nucl. Phys. A 641, 119 (1998).

[20] D. Drechsel, S.S. Kamalov, G. Krein, and L. Tiator, Phys. Rev. D 59, 094021 (1999).

[21] D. Drechsel, S.S. Kamalov, G. Krein, B. Pasquini, and L. Tiator, Nucl. Phys. A 660, 57 (1999).

[22] D. Drechsel, S.S. Kamalov, and L. Tiator, Nucl. Phys. A 645, 145 (1999). Data available at: http://www.kph.uni-mainz.de/MAID/.

[23] X. Ji, C.-W. Kao, and J. Osborne, Phys. Lett. B 472, 1 (2000).

[24] S. S. Kamalov and S. N. Yang, Phys. Rev. Lett. 83, 4494 (1999). 